\title{
Performance of the Gerschgorin Radii and Centers Ratio Detector for Cooperative Spectrum Sensing under Burst Control Channel Errors
}

\author{
Lucas dos Santos Costa, Dayan Adionel Guimarães, and Rausley Adriano Amaral de Souza, Member, IEEE
}

\begin{abstract}
In centralized cooperative spectrum sensing (CSS), cognitive radios (CRs) monitor the spectrum and send the related data to a fusion center (FC) via a control channel in order to more efficiently detect possible idle bands for opportunistic occupation. In practice, such data must be quantized prior to transmission, which can lead to loss of the global spectrum sensing performance due to quantization errors. Additionally, wireless control channel impairments, such as the multipath fading, further contributes to performance degradation. In many researches, the control channel is considered error-free. Even when errors are considered, they usually affect the transmitted symbols independently, that is, the control channel is assumed to be memoryless. Error bursts come from the memory effect of the channel, and are typically found in wireless communications. In this paper we consider the effects of burst errors in the control channel as well as the problem of signal distortion caused by three methods of quantization in centralized CSS with sample fusion. The Gerschgorin Radii and Centers Ratio (GRCR) detector is used as the test statistic for spectrum sensing. Our findings show that: i) the GRCR is robust in the scenarios taken into consideration, ii) the uniform quantization may be preferred in some cases when the control channel is considered perfect, iii) the nonuniform quantization attains better performance under errors in the control channel, and iv) the effect of memory in the control channel may produce, in some situations, performance gains with respect to the memoryless channel, when both have the same average bit error rate.
\end{abstract}

Index Terms-spectrum sensing, memoryless channel, memory channel, Gilbert-Elliott channel, GRCR detector, uniform quantization, nonuniform quantization.

\section{INTRODUCTION}

$\mathbf{T}$ HE rapid expansion of telecommunications services is the primary reason for the current problem of spectrum scarcity and congestion. However, research shows that several spectrum bands are in fact underutilized, since the holders of the exclusive right of use do not do so uninterruptedly and in

L. S. Costa, D. A. Guimarães and R. A. A. de Souza are with the National Institute of Telecommunications (Instituto Nacional de Telecomunicações - Inatel), Santa Rita do Sapucaí, MG, Brazil. E-mail: \{lucass;dayan;rausley\}@inatel.br

This work was supported in part by CNPq under Grant 308365/2017-8, and by RNP, with resources from MCTIC, Grant No. 01250.075413/2018-04, under the Radiocommunication Reference Center (Centro de Referência em Radiocomunicações - CRR) project of the National Institute of Telecommunications (Instituto Nacional de Telecomunicações - Inatel), Brazil.

This article is an invited extended version of [1], Best Paper Award SBrT 2018, which was presented in the XXXVI Simpósio Brasileiro de Telecomunicações e Processamento de Sinais (SBrT 2018), Campina Grande, PB, Brazil, September 16-19, 2018.

Manuscript received March 30, 2019; accepted April 27, 2019.

Digital Object Identifier: 10.14209/jcis.2019.15 every location. Therefore, there are idle bands that could be more efficiently used [2].

The above problem shall be aggravated due to the foreseen unprecedented large number of transceivers in the fifth generation $(5 \mathrm{G})$ of the wireless communication systems, thus demanding a new spectrum access policy. In the current policy, the right to use the spectrum is given by the regulators exclusively to the incumbent licensed user, or primary user (PU), who is free to use the resource in a certain geographic region during the contracted period. The new policy, however, provides for opportunistic access by unlicensed, or secondary users (SUs). In this case, the occupation can be simultaneous to the PUs, provided that any interference is maintained below a pre-established maximum level, or by means of the identification of idle bands to which the SUs have nonsimultaneous access with the PU. To identify idle bands, a reliable spectrum sensing [3] task must be performed by the cognitive radios (CRs) in order to maximize the chance of correctly detecting spectrum allocation opportunities.

Spectrum sensing and final decision on the occupation state of the sensed band can be tasks of a single CR, which is the case in the non-cooperative spectrum sensing model. However, this option is less reliable when compared with the cooperative spectrum sensing (CSS), since cooperation refines the decision by considering data from several CRs that, once in different geographical locations, overcome problems inherent to the lack of cooperation, namely: channel shadowing, hidden terminal and multipath fading [3].

The CSS can be centralized, distributed, or relayassisted [3]. In centralized CSS, each CR sends data via a control channel to the fusion center (FC) where the information is combined in order to arrive at the final decision on the occupation of the sensed band. Two generic types of fusion are commonly defined: decision fusion and data fusion. In decision fusion, each CR makes its own decision and sends it to the FC. In the data fusion model, the data transmitted over the control channel may be samples of the signal received by each CR or other quantity derived from these samples. For example, the fused data can be the eigenvalues of the received signal sample covariance matrix [4], quantities related to the power spectral density of the sensed signal [5], or the energy of the received signal [6].

In both fusion schemes a test statistic is used in a binary hypothesis test to detect the presence or the absence of the PU signal. The test statistic is implemented in each CR in the case of decision fusion, and it is in charge of the FC 
in the case of the data fusion. The FC is always responsible for making the final decision regardless of the fusion scheme adopted. As examples of test statistics, we mention those based on the eigenvalues of the received signal sample covariance matrix [4]: the generalized likelihood ratio test (GLRT), the maximum-minimum eigenvalue detection (MMED), sometimes referred to as eigenvalue ratio detection (ERD), the maximum eigenvalue detection (MED), also called Roy's largest root test (RLRT), and the energy detection (ED), which can also be generated without the computation of eigenvalues. The MED test outperforms others in many scenarios. Nevertheless, because it is semi-blind as the ED, it needs to estimate the noise power and is sensitive to the uncertainty in the estimation, which is usually referred to as noise uncertainty. The same happens with the generalized energy detection (GED) [7] test, which is an evolution of ED.

It should be noted that a sensing technique that is robust against noise uncertainty may be not robust in scenarios where the CRs are subjected to distinct noise powers, which is often referred to as a nonuniform noise situation. Moreover, a robust technique in nonuniform noise scenarios may not be robust under the two effects together when noise levels vary over time, which is known as dynamic noise [8]. As these effects may be present in practical spectrum sensing scenarios, it is necessary to develop techniques that can overcome them.

With this in mind, a new detection technique based on the Gerschgorin circle theorem has recently been proposed. The technique was called the Gerschgorin radii and centers ratio (GRCR) detector [8]. Among other advantages, the GRCR proved to be robust in the simulations involving the practicalappealing scenarios of nonuniform and dynamic noise. In order to provide further analysis of the performances of this new detector in other situations and scenarios, the GRCR is also adopted in the investigations herein.

\section{A. Related works}

Despite the vast literature on CR-based spectrum sensing techniques, there is still a need to consider more realistic scenarios from the standpoint of reliably and flexibly assessing their performances. As an exemplifying initiative, the effect of dynamic and nonuniform noise on the performance of centralized CSS with sample fusion was considered in [8].

Another important aspect of the centralized CSS that has been explored in recent research is the imperfection of the control channel, which is used to exchange control information between the CRs and the FC. Many research works consider this channel perfect, not producing symbol errors in the transmitted data. Moreover, these works do not consider the error produced by the quantization of the information sent when the data fusion approach is adopted. Since quantization errors and symbol errors in the control channel may compromise the performance of the final decisions on the occupation state of given band of interest, it is of the utmost importance that these aspects be taken into account in performance analyzes.

After the publication of [8], the performance of the GRCR detector has been the subject of a few research, for instance [1], where the errors induced by uniform and nonuniform quantizers were investigated under nonuniform noise across the CRs. The results in [1] show that uniform quantization may be preferred when the number of quantization bits is greater than 2, because of the lower complexity and the absence of need for knowing the statistics of the signal to be quantized. As far as quantization is concerned, an scheme based on the Kullback-Leibler divergence is proposed in [9] in order to reduce the amount of data in the control channel. The scheme is able to maintain a performance similar to that achieved with infinite resolution (floating-point representation) with, at best, only 4 quantization bits. The reduction of data traffic in the control channel is also accounted for in [10]. Similarly to [9] the authors of [10] propose a quantization strategy with better results than the other four competing strategies, with the possibility of maintaining the performance close to that obtained with infinite resolution, but using only 3 , 4, 5 and 6 quantization bits. A new nonuniform quantization method is proposed in [11], attaining a performance close to that obtained by uniform quantization. The method applies a variable number of quantization bits, employing higher resolution to specific signal levels. This procedure causes the average number of quantization bits to be reduced at the same time as the performance improves due to nonuniform quantization.

The importance of reducing the amount of data in the control channel and the robustness of the test statistic against the signal distortion due to quantization with low resolution are the focus of [1], [9]-[11]. However, the bit errors produced during the transmission to the FC have not been considered, i.e., the control channel has been considered error-free.

In [12], the bit errors in the control channel are explored in a centralized CSS with decision fusion and data fusion. In the case of data fusion, a quantization scheme with only 2 bits per sample was found to be able to outperform the fusion of decisions in the error-free situation. The research also highlights the performance limitation imposed by errors in the control channel to the two fusion schemes. The effect of these errors are mitigated via an amplify-and-forward method. In [13], a new fusion method is proposed, which also takes into account errors in the control channel. Called on/off signaling, in this scheme CRs report data to the FC only in specific situations defined according to the adopted combination rule at the FC. With the new technique it is possible to achieve performances compatible with those obtained with perfect channel. In addition, it is still possible to reduce the amount of data in the control channel relative to the conventional fusion scheme, while maintaining superior performance. Both quantization errors and bit errors in the control channel are considered in [14], where a fusion technique is proposed to achieve good performance under errors without involving an increase in the amount of data sent to the FC. The results are compared with other fusion schemes in terms of detection performance, amount of information required to perform the spectrum sensing, sensitivity to parameter estimation and computational complexity in parameter optimization. In fact, the technique described in [14] has good performance under errors in the control channel and its computational complexity can be considered low in comparison to the other techniques analyzed. 
In [12]-[14] it is emphasized the importance of considering errors in the control channel when performance analyzes are carried out. However, all these references assume that bit errors are generated by control channels without memory. This means that there is no possibility of errors occurring in the form of bursts in the data sent to the FC.

In summary, [1], [9]-[14] consider quantization errors in some cases, and in others the effects of the quantization errors are added to the errors produced by the control channel. To the best of our knowledge, no analysis has been made assuming burst errors in the information transmitted to the FC. Since this type of error is common in a wireless mobile channel, it is important to consider it in performance analyzes of spectrum sensing schemes. Therefore, in this article, which is an extension of the research presented in [1], we consider the effects of burst errors in the control channel and the problem of signal distortion caused by two nonuniform (nonlinear) quantization methods and a uniform (linear) one, in the centralized CSS with sample fusion, applying the GRCR detector as the test statistic at the FC. Our results show that: i) the GRCR detector is robust in the scenarios analyzed, ii) the uniform quantization may be preferred in some cases when the control channel is assumed to be perfect, iii) the nonuniform quantization attains better performance under errors in the control channel, and iv) the effect of memory in the control channel can generate performance gains with respect to the memoryless channel (especially with uniform quantization) when both are subjected to the same average bit error probability.

\section{B. Contributions and structure of the paper}

The research reported in [1] analyzes the effect of quantization on the performance of the GRCR detector in centralized CSS with sample fusion. The samples in each CR are quantized in three different ways: i) standard uniform quantization, ii) nonuniform quantization under the maximum output entropy (MOE) design criterion, and iii) nonuniform quantization under the minimum mean square error (MMSE) design criterion. Quantized samples are transmitted to the FC through an error-free control channel.

This article covers the investigations reported in [1] using a more detailed approach, and extends them by additionally assuming an erroneous control channel under two models. One model has no memory, meaning that the channel produces independent errors in subsequent bits, which is a characteristic behavior of an additive white Gaussian noise (AWGN) channel. The other model has memory, which means that the channel is capable of producing bit errors that may occur in bursts. The assumption of memory gives an yet more practical appeal to the research, since the memory effect is typical in mobile wireless channels due to multipath fading.

Burst errors are produced by the well-known discrete-time Gilbert-Elliott channel (GEC) model, whose parameters are calculated in a simple way that is consistent with the physical behavior of a mobile wireless channel. When no memory is assumed, a discrete-time binary symmetric channel (BSC) model is used instead.

The results reported herein show that nonuniform quantization attains better performances under errors in the control channel, and that the memory effect of the channel can even bring performance gains in some cases. The performance achieved with the MOE quantizer is the best under errors in the control channel. The opposite is verified with the error-free channel, a case in which the MMSE quantizer outperforms the others.

In summary, the main contributions of this article are:

1) the analysis of the GRCR detector performance under uniform and nonuniform quantization errors in the samples sent to the FC, via error-free control channel, considering different numbers of quantization bits. Part of this analysis is reported in [1];

2) the analysis of the GRCR detector performance under uniform and nonuniform quantization errors affecting the digitized samples sent to the FC, via erroneous control channel, with and without memory, with a fixed number of quantization bits. This contribution is in addition to those in [1];

3) the proposition, as a byproduct, of a simple modeling process to determine the parameters of the GEC model, guaranteeing that a mobile wireless channel is adequately mimicked.

The remainder of this article is organized as follows: in Section II, the system model and the GRCR detector are described. Section III describes the uniform and nonuniform quantization processes. Section IV is devoted to control channel models. The numerical results are presented and discussed in Section V. Finally, the conclusions are drawn in Section VI.

\section{SySTEM MODEL AND THE GRCR DETECTOR}

\section{A. System model}

The action of sensing and deciding on the occupation state of a given frequency band can be modeled mathematically as a binary hypothesis test described by [15]

$$
y(t)= \begin{cases}h x(t)+v(t) & \text { for } \mathcal{H}_{1} \\ v(t) & \text { for } \mathcal{H}_{0}\end{cases}
$$

where $y(t)$ represents the continuous-time received signal, $v(t)$ denotes the Gaussian noise, $x(t)$ represents the signal transmitted by the primary user, $h$ represents the gain of the sensing channel between the primary transmitter and a given receiver, which is usually assumed flat and slow in the context of spectrum sensing, and $\mathcal{H}_{0}$ and $\mathcal{H}_{1}$ denote, respectively, the hypotheses of absence and presence of the primary signal in the sensed band.

The performance of the spectrum sensing can be measured by the probability of detection $P_{\mathrm{d}}=\operatorname{Pr}\left\{\right.$ decision $\left.=\mathcal{H}_{1} \mid \mathcal{H}_{1}\right\}$, and the probability of false alarm $P_{\mathrm{fa}}=\operatorname{Pr}\left\{\right.$ decision $\left.=\mathcal{H}_{1} \mid \mathcal{H}_{0}\right\}$. The probability of detection is the probability of a decision in favor of the presence of the PU signal when it is indeed present. The probability of false alarm is the probability of a decision in favor of the presence of the PU signal when it is in fact absent. In centralized data fusion CSS schemes, it follows that $P_{\mathrm{fa}}=\operatorname{Pr}\left\{T>\gamma \mid \mathcal{H}_{0}\right\}$ and $P_{\mathrm{d}}=\operatorname{Pr}\left\{T>\gamma \mid \mathcal{H}_{1}\right\}$, where $T$ denotes the test statistic used by the FC (the GRCR in the present work), and $\gamma$ is the decision threshold. Performances can be analyzed graphically to facilitate interpretation, by 
means receiver operating characteristic (ROC) curves. A ROC curve gives $P_{\mathrm{fa}}$ versus $P_{\mathrm{d}}$ as they vary by the influence of the variation of the decision threshold $\gamma$.

Under the discrete-time signal model, $N$ complex samples of the signal received by the $m$-th CR, $m=1,2, \ldots, M$, form the vector $\mathbf{y}_{m} \in \mathbb{C}^{N \times 1}$ given by

$$
\mathbf{y}_{m}=\frac{h_{m} \mathbf{x}+\mathbf{v}_{m}}{\sigma_{v_{m}}}
$$

where the scalars $h_{m} \sim \mathbb{C N}(0,1)$ denote the zero-mean complex Gaussian gains of the channels between the primary transmitter and $m$-th CR, modeling a flat and slow Rayleigh fading that is constant during the sensing interval and independent and identically distributed (i.i.d.) across different sensing rounds and CRs. The sensing channel power gains are normalized to 1 , that is, $\mathbb{E}\left\{\left|h_{m}\right|^{2}\right\}=1$, where $\mathbb{E}\{\cdot\}$ is the mathematical expectation operator and $|\cdot|$ is the absolute value of the argument. Still referring to (1), $\mathbf{x} \in \mathbb{C}^{N \times 1}$ represents the signal transmitted by the PU, and is formed by i.i.d. zero-mean complex Gaussian samples with variance $\sigma_{x}^{2}$. This assumption of a Gaussian-distributed PU signal has little impact on the performance of the GRCR detector [8]. Zero mean i.i.d. complex Gaussian samples with variance $\sigma_{v_{m}}^{2}$ form the noise vector $\mathbf{v}_{m} \in \mathbb{C}^{N \times 1}$, which is associated to the thermal noise at the input of the $m$-th CR. Notice that the CRs may be impaired with different noise variances, which characterizes the already-mentioned nonuniform noise model.

It can be noticed in (1) that the noise standard deviation $\sigma_{v_{m}}$ is used to normalize the received signal samples in each CR. We highlight that this is not meant to indicate that this quantity must be explicitly known by each CR in practice. The division by $\sigma_{v_{m}}$ is a simple alternative to model the action of an automatic gain control (AGC), which is commonly present in any receiver, preceding the analog-to-digital conversion. This action can be understood by recalling that the noise variance is approximately equal to the received signal power in the low signal-to-noise ratio (SNR) regime, which is common in the spectrum sensing scenario [16]. Hence, the division by $\sigma_{v_{m}}$ makes the variance of $\mathbf{y}_{m}$ approximately equal to 1, additionally equating the dynamic ranges (in terms of variance) of the samples to be quantized by all CRs.

From all $\left\{\mathbf{y}_{m}\right\}$, firstly assuming no distortion due to quantization and no control channel errors, the received signal sample matrix $\mathbf{Y} \in \mathbb{C}^{M \times N}$ is formed at the $\mathrm{FC}$ as

$$
\mathbf{Y}=\left[\mathbf{y}_{1} \mathbf{y}_{2} \cdots \mathbf{y}_{M}\right]^{\mathrm{T}}
$$

in which the symbol $\mathrm{T}$ stands for the transpose operation.

From $\mathbf{Y}$, the FC computes the sample covariance matrix $\mathbf{R} \in \mathbb{C}^{M \times M}$,

$$
\mathbf{R}=\mathbf{Y} \mathbf{Y}^{\dagger} / N,
$$

in which the symbol $\dagger$ represents the Hermitian (complex conjugate and transpose) operation. Finally, from $\mathbf{R}$ the FC computes the GRCR test statistic, as described in the next subsection, and makes a final decision upon the occupation state of the sensed band.

When bit errors, quantization errors or both are introduced, the real and imaginary samples in $\mathbf{y}_{m}$, as defined in (1), are separately quantized with $b$ bits and digitized before transmission to the FC. Hereafter we denote any quantized variable with a superscript containing the number of quantization bits between parenthesis. For instance, the unquantized $\mathbf{y}_{m}$ becomes the quantized $\mathbf{y}_{m}^{(b)}$, and so on.

\section{B. The GRCR detector}

The Gerschgorin circles theorem [17, p.82] states that the $M$ eigenvalues $\lambda$ of a symmetric matrix of order $M \times M$ are located in the union of $M$ circles (or disks), such that $\left|\lambda-r_{i i}\right| \leq$ $\sum_{j \neq i}\left|r_{i j}\right|$, where $r_{i j}$ is the element in the $i$-th row and $j$-th column of the matrix. In other words, the theorem dictates that the locations of the eigenvalues in the complex plane are inside the region limited by the union of the Gerschgorin circles.

In [8], it was found that the ratio between the sum of the radii and the sum of the centers of the Gerschgorin circles with respect to the sample covariance matrix has different behaviors under the hypotheses $\mathcal{H}_{0}$ and $\mathcal{H}_{1}$. Figure 1 shows the Gerschgorin circles and the corresponding centers derived from a sample covariance matrix $\mathbf{R}$ computed in a CSS scenario containing $M=5 \mathrm{CRs}$, each collecting $N=500$, Figure 1(a), and $N=1500$, Figure 1(b), complex samples of the received signal under an average SNR of $-10 \mathrm{~dB}$. In this case the centers of the circles are always located on the positive part of the real axis, since any covariance matrix is positive semi-definite.
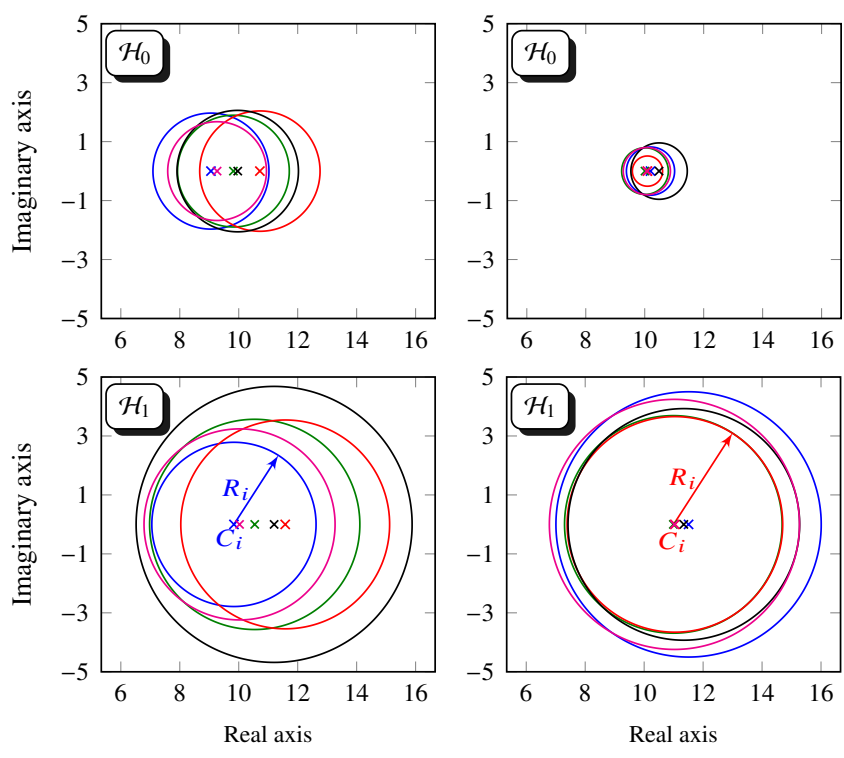

(a) $N=500$

(b) $N=1500$

Fig. 1: Gerschgorin circles under $\mathcal{H}_{0}$ and $\mathcal{H}_{1}$, for a sample covariance matrix R obtained from $M=5$, SNR of $-10 \mathrm{~dB}, N=500$ (a) and $N=1500$ (b). The marks $\times$ are the centers of the circles. This figure is better viewed in color.

Figures 1(a) and 1(b) were both obtained under hypotheses $\mathcal{H}_{0}$ and $\mathcal{H}_{1}$, for $N=500$ and $N=1500$, respectively. It can be seen that, for any of these $N$ values, in fact the results have distinct behaviors across the hypotheses. It can also be noted that the distinct behavior across both hypotheses is more pronounced with the larger $N$. This behavior enables the use of the theorem as test statistic for the detection of the PU signal 
in the context of spectrum sensing. Thus, the test statistic of the GRCR detector is given by the ratio between the sum of the radii and the sum of the centers of the Gerschgorin circles of the sample covariance matrix $\mathbf{R}$, that is,

$$
T_{\mathrm{GRCR}}=\frac{\sum_{i=1}^{M} R_{i}}{\sum_{i=1}^{M} C_{i}},
$$

where $R_{i}=\sum_{j \neq i}\left|r_{i j}\right|$ is the radius and $C_{i}=r_{i i}$ is the center of the $i$-th Gerschgorin circle, for $i, j=1,2, \ldots, M$. If $T_{\mathrm{GRCR}}>\gamma$, the decision at the $\mathrm{FC}$ is made in favor of the presence of the primary signal. Otherwise, if $T_{\mathrm{GRCR}}<\gamma$, the $\mathrm{PU}$ signal is assumed absent in the sensed band.

As quoted above, in practice the samples in $\mathbf{y}_{m}$ must be quantized as part of the digitization prior to transmission. Therefore, it is inevitable that some level of signal distortion will occur as a function of the number of quantization bits of the analog-to-digital converter. When a $b$-bit quantization is applied, the test statistic of the GRCR detector, given in (4), is denoted by $T_{\mathrm{GRCR}}^{(b)}$.

\section{QUANTIZATION METHODS}

\section{A. Uniform quantization}

Figure 2 shows a hypothetical uniform quantization process with $N_{\mathrm{Q}}=2^{b}$ quantization levels. Signal statistics at its input, such as the probability density function (PDF) and the cumulative distribution function (CDF), are in this case completely ignored in the quantization process, and the signal is treated as if it was evenly distributed across its dynamic range.

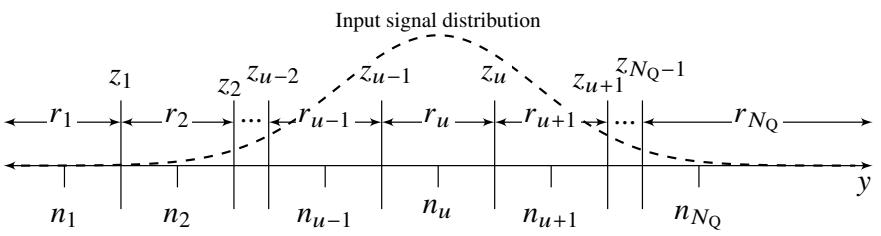

Fig. 2: Hypothetical uniform quantization process.

The quantizer has $N_{\mathrm{Q}}$ output levels $\left\{n_{1}, n_{2}, \ldots, n_{N_{\mathrm{Q}}}\right\}, N_{\mathrm{Q}}-1$ quantization thresholds $\left\{z_{1}, z_{2}, \ldots, z_{N_{Q^{-1}}}\right\}$ equally spaced and $N_{\mathrm{Q}}$ quantization regions, defined as

$$
r_{u}= \begin{cases}\left(\xi, z_{u}\right], & u=1, \\ {\left[z_{u-1}, z_{u}\right],} & 2 \leq u \leq N_{\mathrm{Q}}-1, \\ {\left[z_{u-1},+\infty\right),} & u=N_{\mathrm{Q}} .\end{cases}
$$

In (5) $\xi=0$ exclusively in the quantization of the maximum value in $\mathbf{y}_{m}$, whereas $\xi=-\infty$ in the quantization of the samples in $\mathbf{y}_{m}$.

Considering the signed uniform quantizer described in [18], the $u$-th output level is $n_{u}=-2^{b-f-1}+(u-1) 2^{-f}$. The variable $f$ indicates the number of bits assigned to the fractional part of $n_{u}$. Consequently, $b-f$ is the number of bits assigned to the integer part of $n_{u}$. If $f=0$, all bits are used to quantize the integer part of $n_{u}$. On the other hand, if $f=b$, all bits are used to quantize the fractional part of $n_{u}$. Note that the quantization levels are not symmetric due to the inclusion of the zero as a possible output value, that is, $n_{N_{\mathrm{Q}} / 2+1}=0$. Therefore $\left|n_{1}\right|>$ $n_{N_{\mathrm{Q}}}$. The $u$-th quantization threshold is given by

$$
z_{u}=\left(n_{u}+n_{u+1}\right) / 2, \quad 1 \leq u<N_{\mathrm{Q}} .
$$

In the quantization strategy adopted here, the samples in $\mathbf{y}_{m}$ are normalized by $y_{m_{\max }}$, which is the quantized maximum absolute value (real or imaginary) across the samples of $\mathbf{y}_{m}$. Consequently, the result is the vector $\mathbf{y}_{m}^{\mathrm{n}}=\mathbf{y}_{m} / y_{m_{\max }}^{\left(b_{\max }\right)}$, where $b_{\max }$ indicates the number of bits used to quantize $y_{m_{\max }}$. Using this approach, it is guaranteed that $\left\{-1 \lesssim \mathbf{y}_{m}^{\mathrm{n}} \lesssim 1\right\}$ due to the quantization of $y_{m_{\max }}$ before normalization. Note that we would have $\mathbf{y}_{m}^{\mathrm{n}}$ strictly limited to \pm 1 if $y_{m_{\max }}$ were not quantized before the normalization of $\mathbf{y}_{m}$.

Since $y_{m_{\max }}>0$, its quantization has to be unsigned. Hence, for the uniform quantization of $y_{m_{\max }}$, the $u$-th output level is $n_{u}=(u-1) 2^{-f}$.

The last process before quantizing the received signal samples is to weight $\mathbf{y}_{m}^{\mathrm{n}}$ by $n_{N_{\mathrm{Q}}}=2^{-f}\left(2^{b-1}-1\right)$, yielding $\mathbf{y}_{m}^{\mathrm{nw}}=2^{-f}\left(2^{b-1}-1\right) \mathbf{y}_{m}^{\mathrm{n}}$, which makes the dynamic range of the signal at the quantizer input to be $\left\{-2^{-f}\left(2^{b-1}-1\right) \lesssim \mathbf{y}_{m}^{\mathrm{nw}} \lesssim\right.$ $\left.2^{-f}\left(2^{b-1}-1\right)\right\}$. Thus, as happens in practice, it is possible to control the chances of having samples of the signal outside the dynamic range of the quantizer by controlling $b_{\max }$.

After the quantization of $\mathbf{y}_{m}^{\mathrm{nw}}$ with $b$ bits, $\mathbf{y}_{m}^{\mathrm{nw}^{(b)}}$ and $y_{m_{\max }}^{\left(b_{\max }\right)}$ are sent to the FC through the control channel. At the FC, the quantized and possibly corrupted samples are retrieved according to the operation

$$
\mathbf{y}_{m}^{(b)}=\frac{y_{m m_{\max }}^{\left(b_{\max }\right)}}{2^{-f}\left(2^{b-1}-1\right)} \mathbf{y}_{m}^{\mathrm{nw}^{(b)}} .
$$

Subsequently, the matrix $\mathbf{Y}^{(b)}$ of quantized samples are formed from all $\mathbf{y}_{m}^{(b)}$, analogously to (2), the sample covariance matrix $\mathbf{R}^{(b)}$ is computed from $\mathbf{Y}^{(b)}$, analogously to (3), and the GRCR test statistic $T_{\text {GRCR }}^{(b)}$ is formed from $\mathbf{R}^{(b)}$, analogously to (4). The final decision upon the occupation of the sensed band is then made by comparing $T_{\mathrm{GRCR}}^{(b)}$ with the decision threshold $\gamma$.

\section{B. Nonuniform quantization}

This quantization, to be efficient, requires knowledge of the signal statistics at the quantizer input. According to (1), the samples collected by the CRs prior to the quantization process have a Gaussian distribution with zero mean. Thus, in light of (1), under $\mathcal{H}_{0}$ the PDF and the CDF of the real or imaginary samples to be quantized are respectively given by

$$
\begin{gathered}
f\left(y \mid \mathcal{H}_{0}\right)=e^{-y^{2}} / \sqrt{\pi}, \\
F\left(y \mid \mathcal{H}_{0}\right)=\int_{-\infty}^{y} f\left(y \mid \mathcal{H}_{0}\right) d y=1-\frac{1}{2} \operatorname{erfc}(y),
\end{gathered}
$$

being $\operatorname{erfc}(x)=\frac{2}{\sqrt{\pi}} \int_{x}^{\infty} e^{-t^{2}} d t$ the complementary error function. The variable $y$ denotes any real or imaginary sample collected by the $m$-th CR; indexes were dropped for notational simplicity.

Also in light of (1), under $\mathcal{H}_{1}$ the samples collected by the CRs prior to quantization have a conditioned (on $h_{m}$ ) Gaussian distribution with zero mean. Thus, it follows that

$$
f\left(y \mid \mathcal{H}_{1}\right)=\frac{e^{-y^{2} \sigma_{v_{m}}^{2} /\left(\sigma_{x}^{2}+\sigma_{v_{m}}^{2}\right)}}{\sqrt{\pi\left(\sigma_{x}^{2}+\sigma_{v_{m}}^{2}\right) / \sigma_{v_{m}}^{2}}},
$$




$$
F\left(y \mid \mathcal{H}_{1}\right)=1-\frac{1}{2} \operatorname{erfc}\left(\frac{y \sigma_{v_{m}}}{\sqrt{\sigma_{x}^{2}+\sigma_{v_{m}}^{2}}}\right) .
$$

Figure 3 shows a hypothetical example of a nonuniform quantization process with $N_{\mathrm{Q}}=2^{b}$ quantization levels. The levels are no longer equally spaced, as they are now dependent of the PDF of the input signal. Each level is defined as the center of mass of the PDF in each quantization region, $r_{u}$, as described by (5), with $\xi=-\infty$, that is,

$$
n_{u}=\frac{\int_{r_{u}} y f(y) d y}{\int_{r_{u}} f(y) d y}
$$

where the integral $\int_{r_{u}} y f(y) d y$ corresponds to the expected value of $y$ in the $u$-th quantization region $r_{u}$, being $f(y)=$ $P_{\mathcal{H}_{0}} f\left(y \mid \mathcal{H}_{0}\right)+P_{\mathcal{H}_{1}} f\left(y \mid \mathcal{H}_{1}\right)$ and $P_{\mathcal{H}_{0}}$ e $P_{\mathcal{H}_{1}}$ the probabilities related to PU activity.

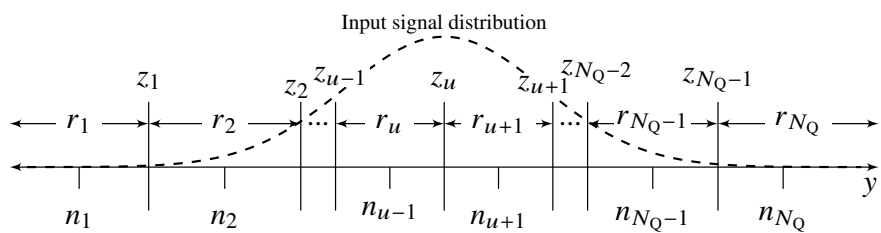

Fig. 3: Hypothetical nonuniform quantization process.

1) Maximum output entropy (MOE) quantizer: The MOE quantizer seeks for quantization thresholds $z_{u}, 1 \leq u<N_{\mathrm{Q}}$, such that the entropy of the output levels is maximized [19]. This is achieved if $\int_{r_{u}} f(y) d y=1 / N_{\mathrm{Q}}$ in each quantization region $r_{u}$. As a consequence, each output level will have probability $p_{u}=\operatorname{Pr}\left\{n_{u}\right\}=1 / N_{\mathrm{Q}}$, and the output entropy becomes $H=-\sum_{u} p_{u} \log _{2}\left(p_{u}\right)=b$ bits.

The quantization thresholds can be obtained by the inverse $\mathrm{CDF}$ of the input signal, i.e.,

$$
z_{u}=F^{-1}\left(\frac{u}{N_{\mathrm{Q}}}\right),
$$

where $F\left(z_{u}\right)=\operatorname{Pr}\left\{y \leq z_{u}\right\}=\int_{-\infty}^{z_{u}} f(y) d y=u / N_{\mathrm{Q}}$ and $F(y)=$ $P_{\mathcal{H}_{0}} F\left(y \mid \mathcal{H}_{0}\right)+P_{\mathcal{H}_{1}} F\left(y \mid \mathcal{H}_{1}\right)$. Subsequently, the output levels of the quantizer are obtained via (12).

2) Minimum mean square error (MMSE) quantizer: In this quantizer, the Lloyd-Max iterative algorithm is used to find the best values of $n_{u}$ so that the MMSE between the input signal $y$ and its quantized version $n_{u}$ is minimized [20]. Since the calculation of $n_{u}$ in (12) requires the knowledge of $z_{u}$ as given by (6), and the calculation of $z_{u}$ requires the knowledge of $n_{u}$, initial values for $n_{u}$ are arbitrarily chosen so that the first calculations of $z_{u}$ are performed. Afterwards, the algorithm recursively calculates new quantization thresholds and output levels until convergence is achieved. Mathematically, the Lloyd-Max algorithm finds the values of $n_{u}$ and $z_{u}$ by solving the unconstrained optimization problem

$$
\operatorname{minimize} \sum_{u=1}^{N_{\mathrm{Q}}} \int_{r_{u}}\left(y-n_{u}\right)^{2} f(y) d y,
$$

being $n_{u}$ and $z_{u}$ the optimization variables. The stopping criterion of the algorithm is based on a maximum target distortion value. If the mean square error is greater than the desired maximum distortion value, new values are calculated for $n_{u}$ and $z_{u}$.

\section{Control Channel Models}

Since the decisions made by the FC upon the occupation state of the sensed band can be affected by errors introduced by the control channel, such errors must be modeled for simulations of analyses. In this section we describe the two models adopted herein, namely: the binary symmetric channel (BSC) without memory, and the Gilbert-Elliott channel (GEC). The former produces independent bit errors, whereas the latter may produce errors in bursts due to the memory effect of the channel. Subsection IV-A and the Subsection IV-B describe the two models, respectively.

\section{A. Binary symmetric channel (BSC)}

Figure 4 illustrates the binary symmetric channel model without memory with error (crossover) probability $P_{\mathrm{e}}$. Thus, the probability of a correctly received bit is $1-P_{\mathrm{e}}$.

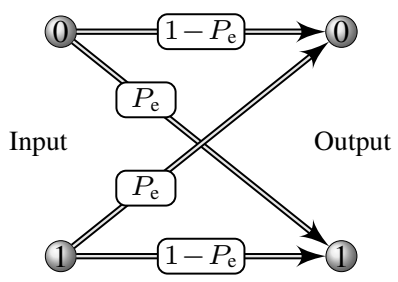

Fig. 4: Memoryless binary symmetric channel (BSC) model.

The BSC model is called binary because the input alphabet is binary. It is said to be symmetric in that a bit ' 0 ' in its input can be turned into a bit ' 1 ' in its output with the same probability that a bit ' 1 ' can be turned into a bit ' 0 '. The errors produced in a given bit is independent of the occurrence or not of an error in the previous bit, which means that the channel has no memory.

The transition matrix of the BSC model is given by

$$
\mathbf{P}=\left[\begin{array}{ll}
p\left(y_{0} \mid x_{0}\right) & p\left(y_{1} \mid x_{0}\right) \\
p\left(y_{0} \mid x_{1}\right) & p\left(y_{0} \mid x_{1}\right)
\end{array}\right]=\left[\begin{array}{cc}
1-P_{\mathrm{e}} & P_{\mathrm{e}} \\
P_{\mathrm{e}} & 1-P_{\mathrm{e}}
\end{array}\right],
$$

where $p\left(y_{0} \mid x_{0}\right)$ is the conditional probability of receiving a bit ' 0 ' given that a bit ' 0 ' was transmitted; the other probabilities are defined analogously.

Figure 5 illustrates the temporal distribution of error events in the BSC and the GEC model during an interval corresponding to 1000 bits. Figure 5(a) depicts the BSC error events, whereas Figure 5(b) and Figure 5(c) refer to the GEC for the average burst lengths $L=5$ and $L=200$ bits, respectively. It can be noticed the evenly-distributed error events in the BSC model, and the burst of errors in the GEC model, with the size of the burst increasing with the increase of $L$.

It is informative to mention that a burst is not necessarily formed by consecutive errors during an entire deep fading. A burst is usually defined as having gaps in-between smaller groups of errors, which is consistent with the physical behavior of the channel: during a deep fading the instantaneous SNR drops drastically, meaning that during this interval the AWGN 
dominates the error events, making them independent, but with higher probability of having groups of consecutive errors.

If the transmissions to the FC apply binary phase-shift keying (BPSK) modulation over a flat (not frequency-selective fading) and slow (approximately constant fading withing a symbol interval) Rayleigh channel, the average probability of bit error is [21]

$$
P_{\mathrm{e}}=\frac{1}{2}\left(1-\sqrt{\frac{\Gamma}{1+\Gamma}}\right)
$$

where $\Gamma=\mathbb{E}\left[\alpha^{2}\right] E_{\mathrm{b}} / N_{0}$ is the average SNR per bit, and where $\alpha$ is the fading envelope, $\mathbb{E}\left[\alpha^{2}\right]$ is the average power gain of the channel, $E_{\mathrm{b}}$ is the average energy per bit, and and $N_{0}$ is the unilateral power spectral density of the AWGN.
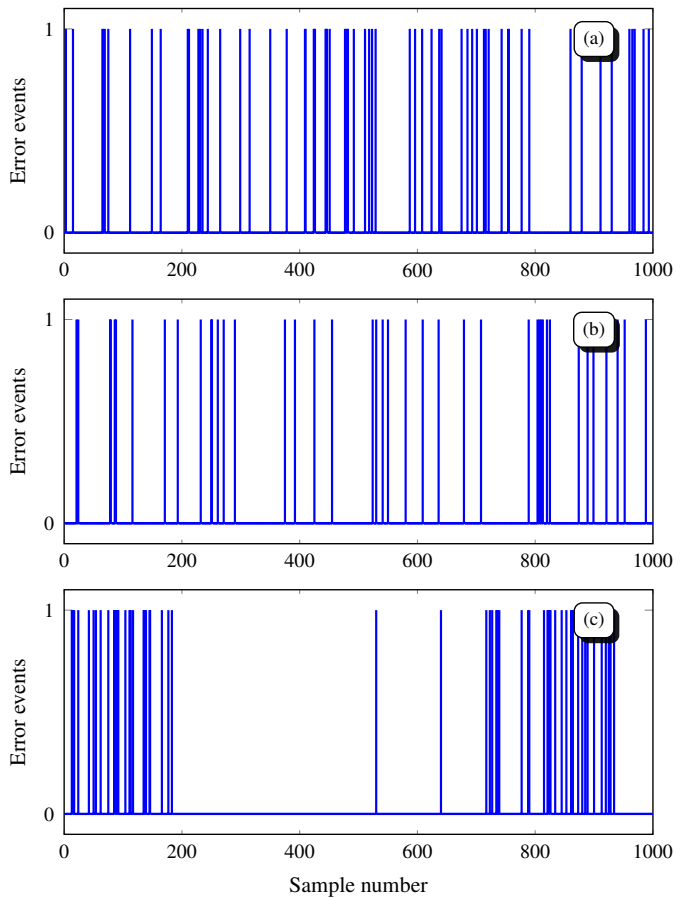

Fig. 5: Temporal error distribution for the BSC (a), and the GEC with different average burst lengths: $L=2$ bits (b), and $L=200$ bits (c).

\section{B. Gilbert-Elliott channel (GEC)}

Figure 6 illustrates the GEC. The model has two states: the good state, which is represented by the letter $\mathrm{G}$, and the bad state, which is represented by the letter B. When in the good state the probability of bit error is given by $P_{\mathrm{G}}$; when in the bad state, this probability is denoted by $P_{\mathrm{B}}$.

The GEC model is an extension of Gilbert's work [22]. In the original Gilbert channel model, errors are only produced in the bad state, that is, $P_{\mathrm{G}}=0$. Thus, in spite of causing errors in bursts, the original model is not flexible in terms of simulations based on physical parameters of an actual wireless channel. Elliott [23] extended the work of Gilbert by allowing errors in both states and possibly with different probabilities. In both Gilbert and Elliott's works, each channel state is defined as an independent BSC model, each with the corresponding error probability (see Figure 6), and with $P_{\mathrm{B}} \gg P_{\mathrm{G}}$. With $P_{\mathrm{B}} \gg P_{\mathrm{G}}$ the chance of having more bits in error in state B is greater than in state $G$, resulting in errors that occur in bursts.

In the model depicted by Figure $6, P_{\mathrm{GG}}$ is the probability of remaining in state $\mathrm{G}$ and $P_{\mathrm{BB}}$ the probability of remaining in state $\mathrm{B}$. The transitions between states occur with probability $P_{\mathrm{GB}}$ from the good to the bad state, and with probability $P_{\mathrm{BG}}$ from the bad to the good state. Hence, the transition matrix of GEC model is

$$
\mathbf{P}=\left[\begin{array}{ll}
P_{\mathrm{GG}} & P_{\mathrm{GB}} \\
P_{\mathrm{BG}} & P_{\mathrm{BB}}
\end{array}\right]=\left[\begin{array}{cc}
P_{\mathrm{GG}} & 1-P_{\mathrm{GG}} \\
1-P_{\mathrm{BB}} & P_{\mathrm{BB}}
\end{array}\right] .
$$

The steady-state probabilities of being in the good and in the bad states are given by $\pi_{\mathrm{G}}=\left(1-P_{\mathrm{BB}}\right) /\left(1-P_{\mathrm{BB}}+1-P_{\mathrm{GG}}\right)$ and $\pi_{\mathrm{B}}=\left(1-P_{\mathrm{GG}}\right) /\left(1-P_{\mathrm{BB}}+1-P_{\mathrm{GG}}\right)$, respectively, and the average bit error probability is calculated generically as [24], [25]

$$
P_{\mathrm{e}}=P_{\mathrm{G}} \pi_{\mathrm{G}}+P_{\mathrm{B}} \pi_{\mathrm{B}}=\frac{P_{\mathrm{G}}\left(1-P_{\mathrm{BB}}\right)+P_{\mathrm{B}}\left(1-P_{\mathrm{GG}}\right)}{1-P_{\mathrm{BB}}+1-P_{\mathrm{GG}}} .
$$

In terms of modeling, the transition probabilities of the GEC are also computed for a given average bit error probability $P_{\mathrm{e}}$, which is also determined from (16) if the control channel is a flat and slow Rayleigh fading channel.

In practice, the length of the burst errors, which is associated to the channel memory depth, is a function of the time over which the received signal remains under fading. Figure 7 helps to understand the memory effect of the channel and the state transitions in the GEC model. Figure 7(a) shows the instantaneous envelope of the signal received by a mobile terminal, and a reference threshold $\rho$ that defines the states good and bad shown in Figure 7(b). During the time over which the signal level remains below the threshold, the error probability increases. Therefore, the longer this time, the greater the average burst error length $L$.

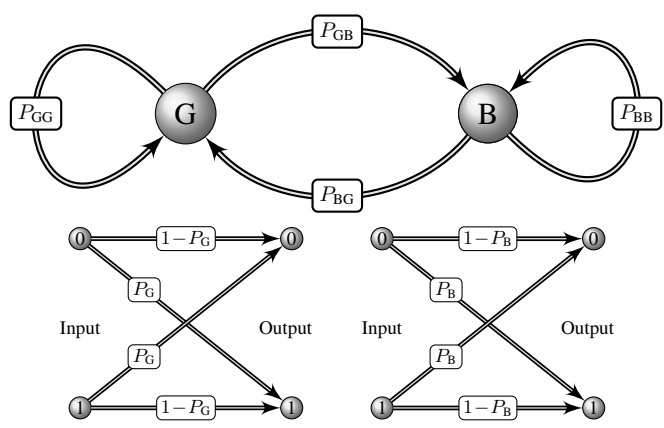

Fig. 6: Gilbert-Elliott channel (GEC) model with memory.

1) Parameterization of the GEC model: The right choice of the GEC model parameters guarantees that the burst error distribution correctly mimics a real mobile wireless control channel. On the other hand, a wrong parameterization not only disconnects the model to the actual channel behavior, but also may lead to inconsistent entries in the channel transition matrix [24], [26]. In order to overcome such problems, firstly some GEC model parameters were estimated by means of a continuous-time waveform channel simulation that takes into account the key physical parameters that influence the statistical behavior of the actual channel [27]. Then, these estimates were used to compute the remaining GEC model 
TABLE I: Key parameters influencing the statistical behavior of the GilbertElliott channel model.

\begin{tabular}{lc}
\hline \multicolumn{1}{c}{ Gilbert-Elliott channel (GEC) model parameters } \\
\hline \hline Carrier frequency & $f_{\mathrm{c}}$ \\
Mobile speed & $v$ \\
Symbol rate & $\mathcal{R}_{\mathrm{s}}$ \\
Light speed & $c=3 \times 10^{8} \mathrm{~m} / \mathrm{s}$ \\
Symbol duration & $T=1 / \mathcal{R}_{\mathrm{s}}$ \\
Maximum Doppler shift & $D_{\max }=v f_{\mathrm{c}} / c$ \\
Reference threshold & $\rho$ \\
Average fading duration & $\tau=\left(e^{\rho^{2}}-1\right) /\left(\rho D_{\max } \sqrt{2 \pi}\right)$ \\
Average number of symbols in $\tau$ & $K=\tau / T$ \\
Average burst length & $L$ \\
\hline \hline
\end{tabular}

parameters in an easy and generic way. The main physical parameters that are related to the statistical behavior of a real mobile wireless fading channel are listed in Table I.

Recalling that the aim of this article is to assess the performance of the GRCR detector when the control channel is subjected to quantization and burst errors, it becomes clear that, at least, different quantization methods and different channel memory depths should be considered. The different quantization methods are those already described in Section III. The different channel memory depths can be achieved by simply setting different average burst lengths $L$. However, it is possible to produce a given $L$ by choosing channels having different combinations of the other parameters given in Table I. In other words, a given $L$ can be achieved for many different sets of the parameters listed in Table I. Thus, the objective of developing a rule for the GEC model parameterization is that of making it simpler and independent, to a certain extent, of the choice of the waveform physical channel parameters.
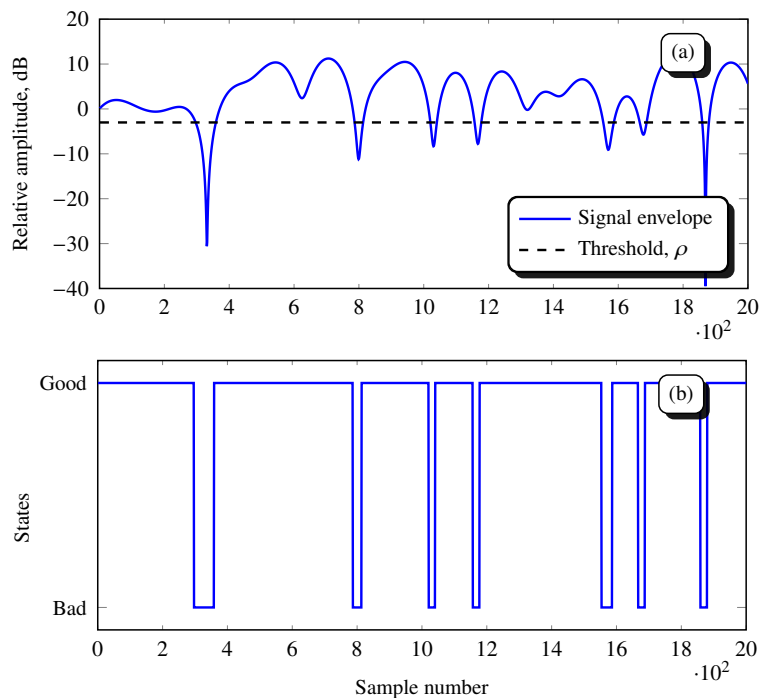

Fig. 7: Reference threshold and instantaneous received signal envelope at a mobile terminal (a), and the states defined according to the threshold (b).

In Table I, $K=\tau / T$ measures the average number of symbols possibly affected during a deep fading; the actual number of affected symbols will depend on the fading statistics itself, and on the AWGN level. However, as $K$ increases, clearly $L$ also increases. Therefore, to simplify matters we make $L=K$. Moreover, since the purpose of the present modeling is to implement computer simulations, we normalize the symbol rate, that is, $\mathcal{R}_{\mathrm{s}}=1 \mathrm{symbol} / \mathrm{s}$, which is a common practice. Hence, the average burst length becomes $L=\tau=K$. Thus, it suffices that $\tau$ be varied so that the burst errors have the desired average length.

With a given $L=\tau$ and $\rho$, the maximum Doppler shift is calculated through $D_{\max }=\left(e^{\rho^{2}}-1\right) /(\rho \tau \sqrt{2 \pi})$. The input of the above-mentioned continuous-time channel simulation are the average SNR per bit, $D_{\max }$, and $\rho=1 / \sqrt{2}$. The estimated parameters are $P_{\mathrm{G}}$ and $P_{\mathrm{B}}$. The above value of $\rho$ was chosen due to the fact that it corresponds to the maximum level crossing rate of the fading envelope, which improves the estimation of the probabilities $P_{\mathrm{G}}$ and $P_{\mathrm{B}}$ for a give simulation execution time. These probabilities are obviously influenced by the SNR, but are also influenced by $D_{\max }$, since a higher Doppler shift means a faster channel fading and, thus, a shorter average fading duration. This shorter fading duration reduces the probability of the channel be in the bad state, which in turn changes $P_{\mathrm{B}}$ and, consequently, $P_{\mathrm{G}}$.

The probability of staying in the bad state, $P_{\mathrm{BB}}$, can be calculated from the average burst error length [28]. Specifically,

$$
P_{\mathrm{BB}}=1-\frac{1}{L}
$$

From this probability, it follows that $P_{\mathrm{BG}}=1-P_{\mathrm{BB}}$ can be determined.

The probability of permanence in the good state, $P_{\mathrm{GG}}$, is derived by means of (18), that is,

$$
P_{\mathrm{GG}}=\frac{P_{\mathrm{B}}-P_{\mathrm{G}}\left(P_{\mathrm{BB}}-1\right)+P_{\mathrm{e}}\left(P_{\mathrm{BB}}-2\right)}{P_{\mathrm{B}}-P_{\mathrm{e}}},
$$

and from (17) $P_{\mathrm{GB}}=1-P_{\mathrm{GG}}$. Since $P_{\mathrm{BB}}=1-1 / L$, as given in (20), the expression for $P_{\mathrm{GG}}$ can be written as

$$
P_{\mathrm{GG}}=1+\frac{P_{\mathrm{G}}-P_{\mathrm{e}}}{L\left(P_{\mathrm{B}}-P_{\mathrm{e}}\right)} \text {. }
$$

\section{NUMERICAL RESULTS}

Computer simulations were carried out using the MATLAB ${ }^{\circledR}$ software, aiming at assessing the performance of the GRCR detector when the control channel is subjected to quantization and burst errors. The performance was analyzed through ROC curves, as well as each corresponding area under the curve (AUC). The AUCs are particularly useful to make it clear any performance difference depicted by close apart ROC curves. Each point on a ROC curve was generated from 150000 Monte Carlo events. In addition, all graphs also contain ideal ROCs, which correspond to the ideal error-free control channel, without quantization distortion (floating-point operation). These curves are identified with the word 'raw' in the graphs.

The CSS scenario consists of a single PU transmitter and $M=9$ CRs. In each sensing period, each CR collects $N=$ 75 or $N=150$ complex samples of the received signal and sends them to the FC via the control channel. The PU signal was transmitted at unit power and the powers of the signal received by the CRs were set so that the average SNRs were configured from $-12 \mathrm{~dB}$ to $-8 \mathrm{~dB}$ in steps of $0.5 \mathrm{~dB}$, yielding an SNR of $-10 \mathrm{~dB}$ when averaged over all CRs. The primary 
TABLE II: Transition matrices as a function of $L$.

\begin{tabular}{|c|c|c|c|c|c|c|}
\hline \multicolumn{7}{|c|}{$\bar{\Gamma}=6 \mathrm{~dB}, P_{\mathrm{e}}=5.3 \times 10^{-2}$ and $\rho=1 / \sqrt{2}$} \\
\hline$L$ & $P_{\mathrm{GG}}$ & $P_{\mathrm{GB}}$ & $P_{\mathrm{BG}}$ & $P_{\mathrm{BB}}$ & $P_{\mathrm{G}}$ & $P_{\mathrm{B}}$ \\
\hline$\overline{2}$ & $\overline{0.499562}$ & 0.500438 & $\overline{0.500000}$ & $\overline{0.500000}$ & $\overline{0.024060}$ & $\overline{0.081912}$ \\
\hline 50 & 0.987027 & 0.0 & 0.020000 & 0.980000 & 0.004170 & 0.128278 \\
\hline 600 & 0.998918 & 0.001082 & 0.001667 & 0.998333 & 0.004135 & 0.128274 \\
\hline 1200 & 0.999465 & 0.000535 & 0.000833 & 0.999167 & 0.004022 & 0.129248 \\
\hline 2400 & 0.999716 & 0.000284 & 0.000417 & 0.999583 & 0.004174 & 0.124598 \\
\hline 4800 & 0.999874 & 0.000125 & 0.000208 & 0.999792 & 0.004473 & 0.133520 \\
\hline
\end{tabular}

user activity was modeled as a Bernouli random variable, with $50 \%$ in the idle state for false alarm counts, and $50 \%$ in the active state for detection counts. Such values correspond to $P_{\mathcal{H}_{0}}=P_{\mathcal{H}_{1}}=0.5$, which were chosen in order to represent the largest uncertainty about the occupancy of the sensed band. It is important to highlight that, if $P_{\mathcal{H}_{0}} \neq P_{\mathcal{H}_{1}} \neq 0.5$, there would be no change in terms of the probability of false alarm, $P_{\mathrm{fa}}$, and the probability of detection, $P_{\mathrm{d}}$, since the statistical power of the detector is not influenced by the PU activity.

The quantization of the samples sent to the FC was made with $b$ bits. When the uniform quantization was adopted, the number of bits assigned to the fractional part of the quantizer output level $n_{u}$ was set to $f=b$, since $\left\{-1 \lesssim \mathbf{y}_{m}^{\mathrm{n}} \lesssim 1\right\}$. The number of bits used to quantize the maximum value (real or imaginary) in $\mathbf{y}_{m}$ was set to $b_{\max }=3$, with $f=1$, since $y_{m_{\max }}>0$. In cases where the erroneous control channel is assumed, the average SNR per bit was set to $\Gamma=6 \mathrm{~dB}$. Thus, the average bit error probability, $P_{\mathrm{e}}$, given by (16), was kept the same for the BSC and the GEC models.

\section{A. Results for the error-free control channel}

In the case of a perfect control channel, the uniform and the nonuniform quantization methods were implemented with $b=4,3$ and 2 bits. Figure 8(a) was generated with $b=4$ quantization bits. Note that with $N=75$ or $N=150$ samples collected by each $\mathrm{CR}$ in each sensing period, the different quantization schemes have practically the same performances, which are also close to the ROCs for the unquantized and error-free transmissions (raw data). This fact reveals that the GRCR detector has sufficient statistical power to operate without high losses even with low analog-to-digital resolution applied to the signal transmitted to the FC. Moreover, since the quantizers have almost the same performances, it is emphasized that the uniform quantization is more attractive in this case due to the lower implementation complexity (not needing to know any statistics of the signal at the quantizer input).

In Figure 8(b), which considers $b=3$ quantization bits, it can be observed that the performances of the quantization schemes are slightly different from each other, and that the sensitivity of the GRCR detector to the quantization resolution starts to become noticeable when compared to Figure 8(a) and to the ideal (raw) ROC. With $N=75$ samples, such differences are slightly larger (see the corresponding AUCs if necessary). From a practical standpoint, however, any of the quantization processes can be adopted when $b=3$, with advantage of the uniform quantizer in terms of complexity, if its slightly lower performance can be considered satisfying.
With $b=2$ bits of resolution, it can be noticed from Figure 8(c) that all quantization methods yield performance losses with respect to the raw ROC, but the uniform quantizer produces the higher performance penalty. The influence of $N$ can again be noticed, with the difference between the raw ROC and the ROCs under quantization being larger with $N=75$ samples. Additionally, it can be concluded that with only $b=2$ bits the nonuniform quantization strategies can yield performances not too far from the one achieved with raw data, with a slight advantage of the quantizer developed under the MMSE criterion over the one developed under the MOE rule.

In terms of overall performance ratings, with $b<4$ quantization bits the results with the MMSE quantizer were the best, followed by the results with the MOE and the uniform quantizers. With $b=4$, all quantization methods attains roughly the same performances, meaning that the uniform quantizer is preferred due to its lower complexity.

The above conclusions are maintained if the system parameters are changed, as can be inferred from [1], where a different set of parameters were adopted and the same conclusions have been drawn.

\section{B. Results for the erroneous control channel}

In the remaining results of this section, quantization was performed with $b=4$ quantization bits, since in this case the performances obtained with the three quantizers are practically the same, and also approximately equal to the performance obtained under the error-free and without quantization transmissions, independent of the different distortion level imposed by each quantizer. Moreover, the number of complex signal samples collect by each CR was fixed in $N=150$.

For the simulations with the GEC model, the average burst error lengths $L$ were varied taking as reference the total number of bits sent to the FC by each $\mathrm{CR}$ in each sensing round. This number is $2 b N+b_{\max }=2 \times 4 \times 150+3=1203$ bits, in the uniform quantization, whereas $2 b N=2 \times 4 \times 150=1200$ bits, in the nonuniform quantization, where the multiplication by 2 is a consequence of applying separate quantization processes for the real and imaginary sample values. Thus, in order to asses the CSS performance with average burst lengths very far below, far below, below, around, above and far above the number of bits transmitted by each CR per sensing round, $L$ was made equal to $2,50,600,1200,2400$ and 4800 bits. These values of $L$ were produced by simply varying the configuration of the Doppler shift in the simulation [27]. For each $L$, a transition matrix $\mathbf{P}$ for the GEC model, as defined in (17), has been computed according to Section IV-B1. The probabilities of the resulting matrices are listed in Table II 
according to the value of $L$, for $\Gamma=6 \mathrm{~dB}, P_{\mathrm{e}}=5.3 \times 10^{-2}$ and $\rho=1 / \sqrt{2}$. The table also gives $P_{\mathrm{G}}$ and $P_{\mathrm{B}}$ for each $L$. Independent GEC models were applied to the transmissions performed by each of the $M=9$ CRs.
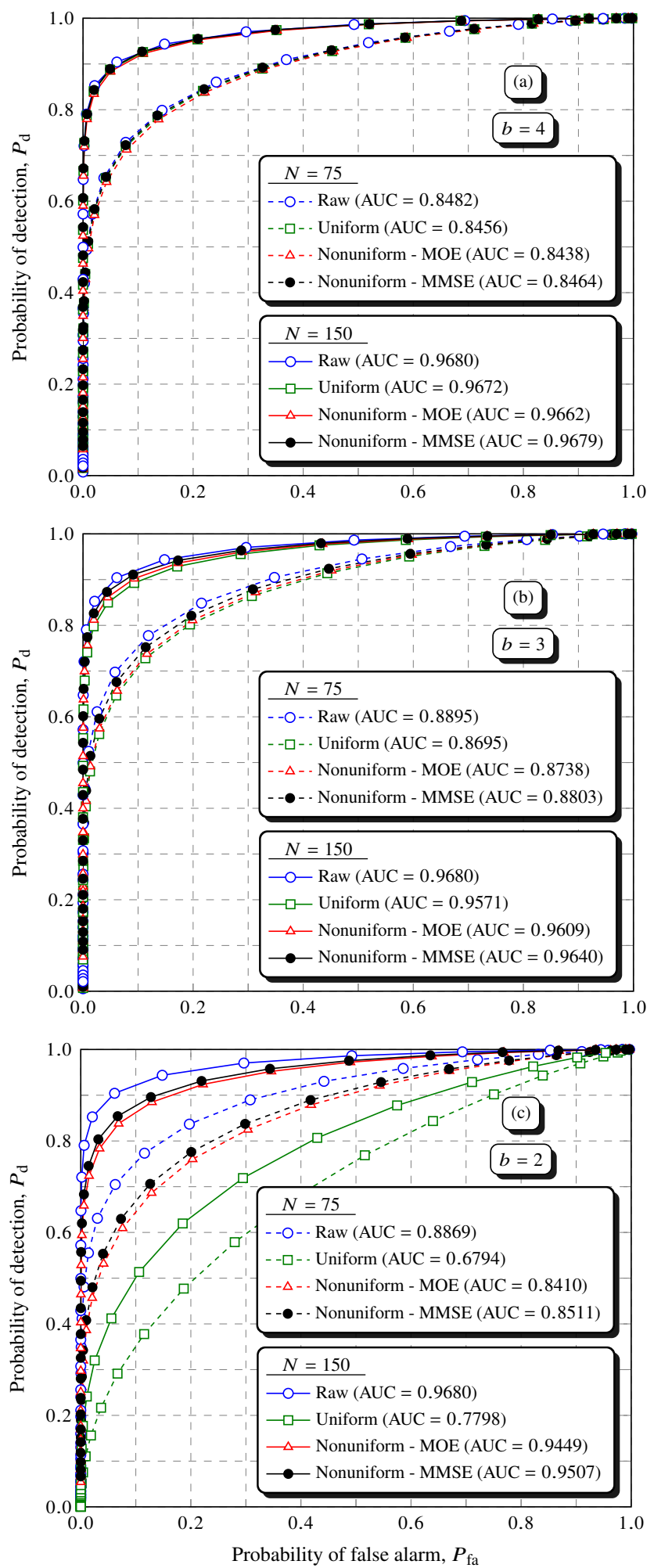

Fig. 8: ROC curves for the error-free control channel with uniform and nonuniform quantization, with $b=4$ (a), $b=3$ (b) and $b=2$ (c) bits, and with $N=75$ and $N=150$ samples.

Figure 9 presents the results for the erroneous control channel with the BSC and the GEC models for different values of $L$. Curves for the error-free channel (EFC) and no quantization (raw data), as well as curves for the EFC with $b=4$ quantization bits were added for comparison purposes.

Figure 9(a) considers $L=2$, which yields approximately the same error statistics as the BSC model. Indeed, the performances obtained with the GEC and the BSC models are practically equal to one another for each of the three quantizers (uniform, nonuniform under MOE rule, and nonuniform under MMSE rule), which is an expected outcome.

In all graphs of Figure 9 it can be seen that the performance achieved with all quantizers in the error-free channel closely approximates the raw data performance, in which the channel is error-free and applies no quantization (floating-point operation), a result already reported in Figure 8(a).

The graphs (b) to (f) in Figure 9 show that the performances obtained with the nonuniform quantizer designed under the MMSE rule improves slightly as $L$ increases, the same happening, but more noticeably, with the uniform quantizer. This can be inferred by observing the corresponding ROCs departing from the ones considering the BSC model. Thus, it can be concluded that in some cases the effect of memory in the control channel can even produce performance gains to the centralized CSS with the GRCR detector. It remains an open problem to justify this behavior, since it is not clear how the memory depth relates to the effects of the quantization errors. The performances obtained with the nonuniform MOE quantizer have shown that it is immune to the channel memory depth.

In general, errors produced in the control channel can significantly affect the performances of the GRCR detector, especially if uniform quantization is adopted. However, the detector is capable of offering attractive performances considering the high average bit error probability of the control channel, which is $P_{\mathrm{e}}=5.3 \times 10^{-2}$ for $\Gamma=6 \mathrm{~dB}$.

The performances with the MOE quantizer proved to be the most robust under errors in the control channel. In addition to this advantage, it is emphasized that the MOE quantizer has a smaller implementation complexity, fewer operations and, consequently, less processing time than the MMSE. In terms of implementation complexity, the uniform quantizer is the best, but the performances have been the most sensitive to the control channel errors.

\section{Conclusions}

This paper considered a centralized cooperative spectrum sensing with sample fusion, exploring the performance of the recently proposed Gerschgorin radii and centers ratio (GRCR) detector, under independent and burst errors in the control channel transmissions, as well as under the effect of different signal quantization methods and resolutions.

Under the error-free control channel, the GRCR proved to be quite robust to signal distortion due to quantization. In addition, it has been demonstrated that uniform quantization may be preferred when the number of bits is greater than $b=2$ due to the attractive performance results and the unneeded statistics of the input signal. With $b=2$, the performance 

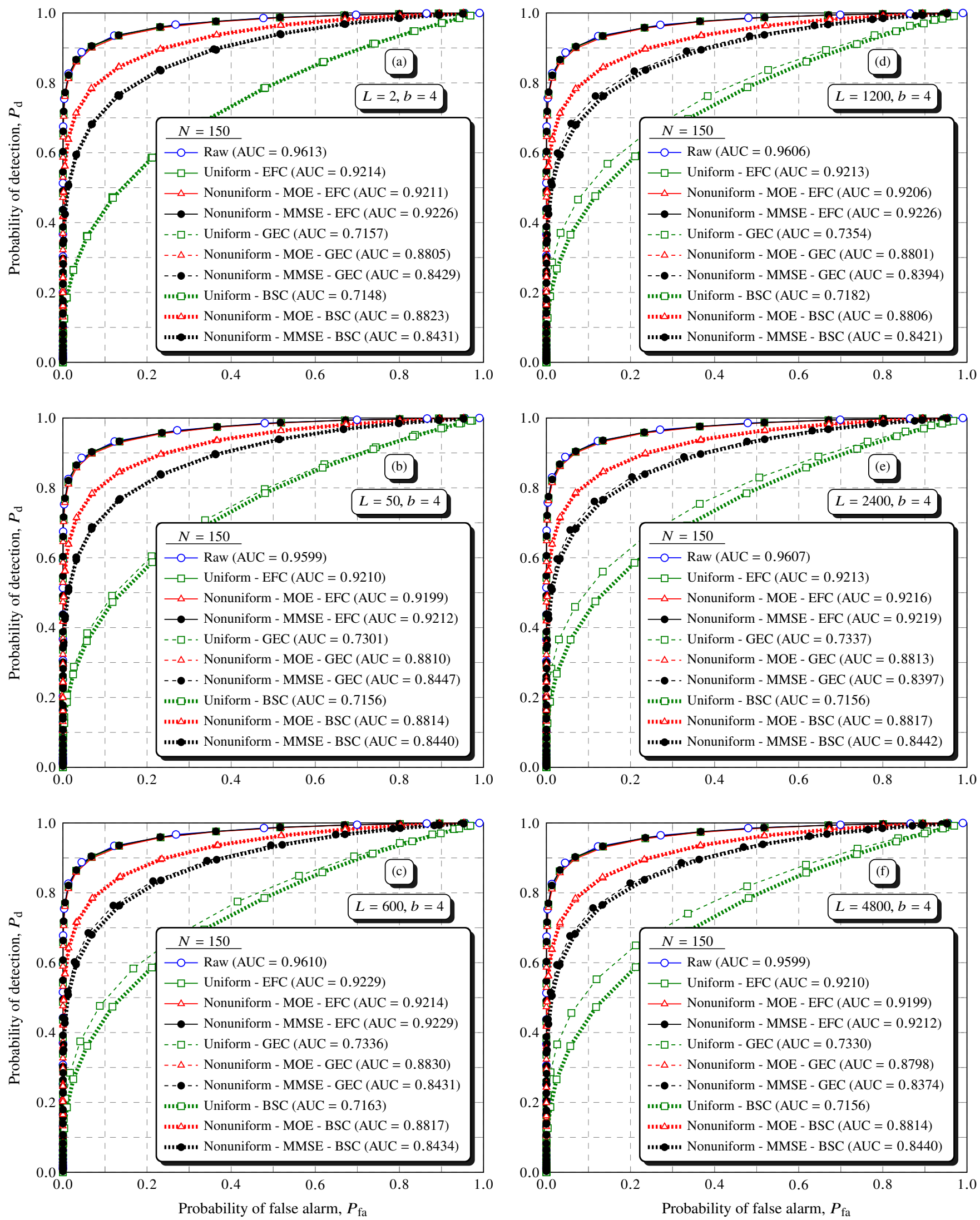

Fig. 9: ROC curves for erroneous control channel with uniform and nonuniform quantization, with $b=4$ bits, $N=150$ samples and average burst error lengths $L=2$ (a), $L=50$ (b), $L=600$ (c), $L=1200$ (d), $L=2400$ (e) and $L=4800$ (f) bits. This figure is better viewed in color and zoomed in. 
suffers great losses if the uniform quantization is adopted; the nonuniform quantizers yielded quite superior performances in this case.

It has been shown that quantization errors and an erroneous control channel can significantly affect the CSS performance in comparison to the cases in which the error-free control channel with infinite resolution (floating-point operation) is considered. It has been also observed that, in some cases, the memory effect of the GEC model can bring performance gains as the memory depth increases. The nonlinear quantizer developed under the MOE rule showed better performances in the erroneous control channel, followed by the MMSE-based nonlinear quantizer, and by the uniform quantizer.

\section{REFERENCES}

[1] L. S. Costa, D. A. Guimarães, and R. A. A. de Souza, "Original title in Portuguese: Influência da quantização no sensoriamento espectral via teorema dos círculos de gerschgorin,” in XXXVI Brazilian Telecommun. Symp., SBrT'18, Sep. 2018.

[2] Y. Chen and H. Oh, "A survey of measurement-based spectrum occupancy modeling for cognitive radios," IEEE Commun. Surveys Tuts., vol. 18, no. 1, pp. 848-859, Firstquarter 2016. doi: 10.1109/COMST.2014.2364316

[3] I. F. Akyildiz, B. F. Lo, and R. Balakrishnan, "Cooperative spectrum sensing in cognitive radio networks: A survey," Physical Commun., vol. 4, no. 1, pp. 40 - 62, Mar. 2011. doi: https://doi.org/10.1016/j.phycom.2010.12.003. [Online]. Available: http://www.sciencedirect.com/science/article/pii/S187449071000039X

[4] B. Nadler, F. Penna, and R. Garello, "Performance of eigenvalue-based signal detectors with known and unknown noise level," in 2011 IEEE Int. Conf. on Commun. (ICC), Jun. 2011. doi: 10.1109/icc.2011.5963473. ISSN $1938-1883$ pp. 1-5.

[5] R. C. D. V. Bomfin, R. A. A. de Souza, and D. A. Guimarães, "Circular folding cooperative power spectral density split cancellation algorithm for spectrum sensing," IEEE Commun. Lett., vol. 21, no. 2, pp. 250-253, Feb. 2017. doi: 10.1109/LCOMM.2016.2630700

[6] S. Nallagonda, S. K. Bandari, S. D. Roy, and S. Kundu, "Performance of cooperative spectrum sensing with soft data fusion schemes in fading channels," in 2013 Annu. IEEE India Conf. (INDICON), Dec. 2013. doi: 10.1109/INDCON.2013.6725924. ISSN 2325-940X pp. $1-6$.

[7] Y. Chen, "Improved energy detector for random signals in Gaussian noise," IEEE Trans. Wireless Commun., vol. 9, no. 2, pp. 558-563, Feb. 2010. doi: 10.1109/TWC.2010.5403535

[8] D. A. Guimarães, "Robust test statistic for cooperative spectrum sensing based on the Gerschgorin circle theorem," IEEE Access, vol. 6, pp. 2445-2456, 2018. doi: 10.1109/ACCESS.2017.2783443

[9] H. Vu-Van and I. Koo, "A quantization scheme based on KullbackLeibler divergence for cooperative spectrum sensing in cognitive radio," The Third Int. Conf. on Advances in Cognitive Radio, pp. 44-47, Apr. 2013.

[10] Y. Fu, F. Yang, and Z. He, "A quantization-based multibit data fusion scheme for cooperative spectrum sensing in cognitive radio networks," Sensors, vol. 18, no. 2, 2018. doi: 10.3390/s18020473. [Online]. Available: http://www.mdpi.com/1424-8220/18/2/473

[11] A. Chakraborty, J. S. Banerjee, and A. Chattopadhyay, "Non-uniform quantized data fusion rule for data rate saving and reducing control channel overhead for cooperative spectrum sensing in cognitive radio networks," Wireless Personal Commun., vol. 104, no. 2, pp. 837 851, Jan. 2019. doi: 10.1007/s11277-018-6054-1. [Online]. Available: https://doi.org/10.1007/s11277-018-6054-1

[12] H. Sakran and M. Shokair, "Hard and softened combination for cooperative spectrum sensing over imperfect channels in cognitive radio networks," Telecommun. Syst., vol. 52, no. 1, pp. 61-71, Jan. 2013. doi: 10.1007/s11235-011-9467-7. [Online]. Available: https://doi.org/10.1007/s11235-011-9467-7

[13] S. Bae and H. Kim, "Robust cooperative sensing with on/off signaling over imperfect reporting channels," IEEE Trans. Ind. Informat., vol. 12, no. 6, pp. 2196-2205, Dec. 2016. doi: 10.1109/TII.2016.2607124

[14] I. Hwang and J. W. Lee, "Cooperative spectrum sensing with quantization combining over imperfect feedback channels," IEEE Trans. Signal Process., vol. 65, no. 3, pp. 721-732, Feb. 2017. doi: 10.1109/TSP.2016.2626251
[15] A. Ghasemi and E. S. Sousa, "Collaborative spectrum sensing for opportunistic access in fading environments," in First IEEE Int. Symp. on New Frontiers in Dynamic Spectrum Access Networks, 2005. DySPAN 2005., Nov. 2005. doi: 10.1109/DYSPAN.2005.1542627 pp. 131-136.

[16] IEEE Standard for information technology- Local and metropolitan area networks-Specific requirements-Part 22: Cognitive Wireless RAN Medium Access Control (MAC) and Physical Layer (PHY) specifications: Policies and procedures for operation in the TV Bands, IEEE Std., Jul. 2011.

[17] J. W. Demmel, Applied numerical linear algebra. Philadelphia, PA, USA: Society for Industrial and Applied Mathematics, 1997, vol. 56. ISBN 0-89871-389-7

[18] MathWorks. (2016, Dec.) PDF documentation for fixed-point designer. FPTUG.pdf. [Online]. Available: https://www.mathworks.com/help/pdf_ doc/fixedpoint/index.html?s_cid=doc_ftr

[19] D. Messerschmitt, "Quantizing for maximum output entropy," IEEE Trans. Inf. Theory, vol. 17, no. 5, pp. 612-612, Sep. 1971. doi: 10.1109/TIT.1971.1054681

[20] J. Max, "Quantizing for minimum distortion," IRE Trans. Inf. Theory, vol. 6, no. 1, pp. 7-12, Mar. 1960. doi: 10.1109/TIT.1960.1057548

[21] D. A. Guimarães, Digital Transmission: A Simulation-Aided Introduction with VisSim/Comm, 1st ed. Springer Publishing Company, Incorporated, 2009. ISBN 3642013589, 9783642013584

[22] E. N. Gilbert, "Capacity of a burst-noise channel," The Bell Syst. Tech. $J$. , vol. 39, no. 5, pp. 1253-1265, Sep. 1960. doi: 10.1002/j.15387305.1960.tb03959.x

[23] E. O. Elliott, "Estimates of error rates for codes on burst-noise channels," The Bell Syst. Tech. J., vol. 42, no. 5, pp. 1977-1997, Sep. 1963. doi: 10.1002/j.1538-7305.1963.tb00955.x

[24] G. Hasslinger and O. Hohlfeld, "The Gilbert-Elliott model for packet loss in real time services on the internet," in 14th GI/ITG Conf. Measurement, Modelling and Evalutation of Computer and Commun. Syst., Mar. 2008, pp. 1-15.

[25] J. R. Yee and E. J. Weldon, "Evaluation of the performance of errorcorrecting codes on a Gilbert channel," IEEE Trans. Commun., vol. 43, no. 8, pp. 2316-2323, Aug. 1995. doi: 10.1109/26.403764

[26] S. D. Morgera and F. Simard, "Parameter estimation for a burst-noise channel," in [Proc.] ICASSP 91: 1991 Int. Conf. on Acoust., Speech, and Signal Process., Apr. 1991. doi: 10.1109/ICASSP.1991.150634. ISSN 1520-6149 pp. 1701-1704 vol.3.

[27] D. A. Guimarães. (2019, Mar.) Estimation of the probabilities in the good and bad states of a Gilbert-Elliot channel model using the VisSim/Comm software. [Online]. Available: https://www.dropbox.com/ s/qv9w605q8tcawf2/BPSK_under_fading_for_Gilbert_Elliot.vsm?dl=0

[28] A. Willig, "A new class of packet- and bit-level models for wireless channels," in The 13th IEEE Int. Symp. on Personal, Indoor and Mobile Radio Commun., vol. 5, Sep. 2002. doi: 10.1109/PIMRC.2002.1046581 pp. 2434-2440 vol.5.

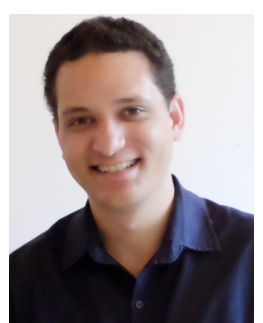

Lucas dos Santos Costa was born in Brazil in 1982. $\mathrm{He}$ received the B.S.E.E. and the M.Sc. degrees from the National Institute of Telecommunications, INATEL, in 2011 and 2014, respectively. Since 2015 he has been working at the Reference Center for Radiocommunications, whose research is concentrated on the fifth-generation (5G) of wireless and mobile communications. His main area of interest is related to digital communication systems and, specifically, he has been working with spectrum sensing for cognitive radio applications. 


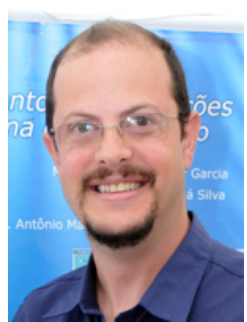

Dayan Adionel Guimarães was born in Carrancas, MG, Brazil, on March 01, 1969. He holds the titles: Electronics Technician (1987), Electrical Engineer (1994), Specialist in Data Communication Engineering (2003), Specialist in Human Resources Management (1996), Master in Electrical Engineering (1998) and Doctor in Electrical Engineering (2003). In 2010 he attended a Pos-Doctoral internship at Federal University of Santa Catarina (UFSC) to study Convex Optimization. From 1988 to 1993 he developed equipment for Industrial Instrumentation and Control, and also occupied the positions of Manufacturing Supervisor and Product Engineering Supervisor at SENSE Sensors and Instruments. Since January 1995 he is a Researcher and a Lecturer with the National Institute of Telecommunications, INATEL, where for eight years he was responsible for the structure that supports practical teaching activities for the Electrical Engineering undergraduate course. His research includes the general aspects of fixed and mobile wireless communications, specifically digital transmission, spectrum sensing for cognitive radio, radio propagation, and convex optimization and signal processing applied to communications.

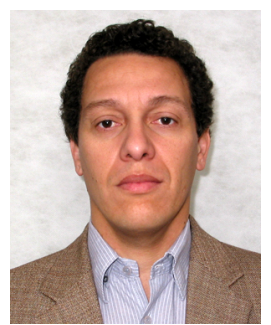

Rausley Adriano Amaral de Souza was born in Brazil in 1972. He received the B.S.E.E. and the M.Sc. degrees from the National Institute of Telecommunications, INATEL, in 1994 and 2002, respectively, and the Ph.D. degree from School of Electrical and Computer Engineering of the State University of Campinas, UNICAMP, Brazil. From 1995 to 2001, he worked as a Purchase Manager at Leucotron Equipamentos Ltda. He joined the National Institute of Telecommunication, INATEL, in 2002, where he is a Full Professor. He is presently a Researcher of the CNPq. Since 2015 he has been working at the Radiocommunication Reference Center, whose researches are concentrated on the fifth-generation (5G) systems. He is a Member of the Brazilian Telecommunications Society (SBrT) and is a Member of the IEEE. His general research interests include wireless communications, fading channel modeling and simulation, diversity systems, and spectrum sensing in cognitive radio systems. 